\title{
ON THE SPARSITY OF REPRESENTATIONS OF RINGS OF PURE GLOBAL DIMENSION ZERO
}

\author{
BIRGE ZIMMERMANN-HUISGEN AND WOLFGANG ZIMMERMANN
}

\begin{abstract}
It is shown that the rings $R$ all of whose left modules are direct sums of finitely generated modules satisfy the following finiteness condition: For each positive integer $n$ there are only finitely many isomorphism types of (a) indecomposable left $R$-modules of length $n$; (b) finitely presented indecomposable right $R$-modules of length $n ;(\mathrm{c})$ indecomposable right $R$-modules having minimal projective resolutions with $n$ relations. Moreover, our techniques yield a very elementary proof for the fact that the presence of the above decomposability hypothesis for both left and right $R$-modules entails finite representation type.
\end{abstract}

\section{INTRODUCTION}

It is an old problem, rooted in work of Koethe (1935 [12]) and Cohen and Kaplansky (1951 [6]), to determine the rings all of whose left modules have direct-sum decompositions into components of "manageable" size (e.g., finitely generated or cyclic or indecomposable). While the commutative case has been settled completely (all decomposability requirements lead to the artinian principal ideal rings in that case; see $[12,6,8,17])$, the noncommutative situation is far from being understood. In particular, it is open whether the rings that qualify need to be of finite representation type in general (i.e., whether they need to be artinian and endowed with only finitely many isomorphism classes of finitely generated indecomposable modules on either side).

Our primary goal here is to show that the rings at stake come "close" to having finite representation type. More precisely, we will prove the following: If $R$ is a ring all of whose left modules are direct sums of finitely generated modules, then

(FIN) $\quad R$ admits only finitely many isomorphism types of indecomposable left $R$-modules of length $n$ for each positive integer $n$.

Received by the editors July 21,1988 and, in revised form, October 20,1988. The results of this paper were presented at the AMS Regional Meeting 842 in Las Cruces, New Mexico, April 8, 1988.

1980 Mathematics Subject Classification (1985 Revision). Primary 16A35, 16A46, 16A48, $16 \mathrm{~A} 64$.

The research of the first author was partially supported by a grant from the National Science Foundation. 
Moreover, we will obtain a weak analogue of (FIN) on the other side: Namely, a ring $R$ as above admits only finitely many isomorphism types of indecomposable finitely presented right $R$-modules of length $n$ for each positive integer $n$.

Thus the question of whether rings of left pure global dimension zero have finite representation type can be viewed as a restricted variant of the second Brauer Thrall conjecture. (Note that Ringel [13] has shown that the second Brauer Thrall conjecture does not hold for arbitrary artinian rings with infinite center.)

How does this result fit into the context of facts already established in the area?

Recently, Zayed [19] observed that the following dichotomy holds for the rings $R$ whose left modules are direct sums of finitely generated modules: Either they satisfy (FIN) or else

(INF) there are infinitely many integers, each of which arises as the length of infinitely many nonisomorphic indecomposable left $R$-modules.

Our result decides the issue: (INF) cannot occur for the rings $R$ under consideration. (Incidentally, it is not known whether every artinian ring satisfies either (FIN) or (INF), although Smalø [15] proved that this dichotomy does hold for all Artin algebras.)

Going further back: Barring the condition that all left modules split into cyclic summands, all "reasonable" global decomposability requirements still describe the same class of rings in the noncommutative situation. In fact, the following statements are equivalent (see [10, 20, 22]):

(I) Each left $R$-module is a direct sum of finitely generated modules.

(II) There exists a cardinal number $\aleph$ such that each left $R$-module is a direct sum of $\aleph$-generated modules.

(III) Each left $R$-module is a direct sum of indecomposable modules.

(IV) $R$ has left pure global dimension zero (i.e., each pure inclusion of left $R$-modules splits).

Moreover, it is well known that the rings having vanishing left and right pure global dimension are precisely those of finite representation type (see [14, 7 and 1]). Concerning the strength of the one-sided condition (IV): Cutting the class of rings under consideration back to Artin algebras, Auslander proved that (IV) guarantees finite representation type (see [2]). The result we prove here can be seen as a continuation of this line, even though our methods are quite different.

The presentation of our argument is almost self-contained. Furthermore, our techniques yield as a by-product a very elementary proof for the fact that vanishing of the left and the right pure global dimension entails finite representation type. 


\section{THE TOOL KIT}

(a) The Auslander-Bridger transpose. Often the lack of helpful self-dualities of the ring $R$ (in particular, the lack of Morita dualities $R$-mod $\rightarrow \bmod -R$ ) forces one to build "crutches" when it comes to shifting information from left to right. A very effective "crutch" of this kind is the Auslander-Bridger transpose [3]. Whenever $R$ is semiperfect, this transpose yields in particular a bijective correspondence between the finitely presented indecomposable left $R$-modules and the finitely presented indecomposable right $R$-modules. We will present a brief sketch, referring to Warfield's presentation of the subject [18] for details.

Let $R$ be semiperfect. Then each finitely generated $R$-module has a unique decomposition (up to isomorphism) of the form $P \oplus N$, where $P$ is projective and $N$ has no nonzero projective direct summands. Given a finitely presented module $M$ (left or right) without nonzero projective summands, let

$$
Q \rightarrow P \rightarrow M \rightarrow 0
$$

be a minimal projective resolution of $M$, and denote by $P^{*}$ resp. $Q^{*}$ the $R$-dual of $P$ resp. $Q$ (i.e., $P^{*}=\operatorname{Hom}_{R}(P, R)$ ). Then the cokernel $\operatorname{Tr}(M)$ of the induced map $P^{*} \rightarrow Q^{*}$ is in turn free of nonzero projective summands, and $P^{*} \rightarrow Q^{*} \rightarrow \operatorname{Tr}(M) \rightarrow 0$ is a minimal projective resolution of $\operatorname{Tr}(M)$. In this setup we have $\operatorname{Tr}(\operatorname{Tr}(M)) \cong M$. Moreover, it is important (and obvious) that $\operatorname{Tr}$ commutes with finite direct sums. To extend $\operatorname{Tr}$ to arbitrary finitely presented modules, suppose $M$ is a finitely presented left module, say $M \cong$ $\operatorname{Re}_{1} \oplus \cdots \oplus \operatorname{Re}_{k} \oplus N$, where $N$ is without nonzero projective summands. Set

$$
\operatorname{Tr}(M)=e_{1} R \oplus \cdots \oplus e_{k} R \oplus \operatorname{Tr}(N),
$$

and supplement this convention by its right-hand analogue.

While $\mathrm{Tr}$ is not an honest duality in that there is no canonical choice of a map $\operatorname{Tr}(f): \operatorname{Tr}\left(M_{2}\right) \rightarrow \operatorname{Tr}\left(M_{1}\right)$ for a map $f: M_{1} \rightarrow M_{2}$ between finitely presented $R$-modules in general, we do have the following. Suppose that $M_{1}$ and $M_{2}$ are $R$-modules without nonzero projective summands and that

$$
Q_{i} \rightarrow P_{i} \rightarrow M_{i} \rightarrow 0
$$

is a minimal projective resolution for $i=1,2$. Then $f: M_{1} \rightarrow M_{2}$ can be lifted to yield maps $g: P_{1} \rightarrow P_{2}$ and $h: Q_{1} \rightarrow Q_{2}$; the $R$-duals $h^{*}: Q_{2}^{*} \rightarrow Q_{1}^{*}$ and $g^{*}: P_{2}^{*} \rightarrow P_{1}^{*}$ in turn give rise to a homomorphism $\operatorname{Tr}(f): \operatorname{Tr}\left(M_{2}\right) \rightarrow$ $\operatorname{Tr}\left(M_{1}\right)$. It is readily verified that for arbitrary choices of $\operatorname{Tr}(f)$ formed in this manner, the following hold:

(i) $f$ is an isomorphism if and only if $\operatorname{Tr}(f)$ is an isomorphism.

(ii) $f$ can be factored through a projective module if and only if the same is true for $\operatorname{Tr}(f)$.

(iii) If $M_{1}, M_{2}, M_{3}$ are finitely presented modules without nonzero projective direct summands and if $M_{1} \stackrel{f}{\rightarrow} M_{2} \stackrel{g}{\rightarrow} M_{3}$ are linking homomorphisms, then $\operatorname{Tr}(f) \cdot \operatorname{Tr}(g)$ is an "eligible" choice for $\operatorname{Tr}(g \cdot f)$ (where eligibility is as explained above). 
The crucial observation to enter the picture later is the following lemma (compare [7] for a related result given without proof).

Lemma Tr. Suppose that $R$ is semiprimary, and let $M_{1} \stackrel{f_{1}}{\rightarrow} M_{2} \stackrel{f_{2}}{\rightarrow} M_{3} \rightarrow \cdots$ be a chain of homomorphisms between finitely presented $R$-modules $M_{n}$ without nonzero projective summands such that $f_{n} \cdots f_{1} \neq 0$ for all $n$. Then there exists an index $m$ with the property that for all choices of $\operatorname{Tr}\left(f_{i}\right)$ (as outlined above), all consecutive compositions of maps along the sequence

$$
\cdots \rightarrow \operatorname{Tr}\left(M_{m+2}\right) \stackrel{\operatorname{Tr}\left(f_{m+1}\right)}{\longrightarrow} \operatorname{Tr}\left(M_{m+1}\right) \stackrel{\operatorname{Tr}\left(f_{m}\right)}{\longrightarrow} \operatorname{Tr}\left(M_{m}\right)
$$

are nonzero as well.

Proof. Suppose that the $M_{n}$ are left modules. In view of remarks (ii) and (iii) above it suffices to establish the existence of an index $m$ such that for all $n>m$ the composition $f_{n} \cdots f_{m}$ cannot be factored through a projective module. If this claim were false, then after cutting off an initial segment and telescoping the remainder of the sequence, we may assume that there exist factorizations

$$
M_{1} \underset{g_{1} P_{P_{1}} / h_{1}}{\stackrel{f_{1}}{\longrightarrow}} M_{2} \underset{P_{2} / h_{2}}{\stackrel{f_{2}}{\longrightarrow}} M_{3} \stackrel{g_{3}}{\longrightarrow} \cdots
$$

through projective modules $P_{n}$. Since the $M_{n}$ 's are without nontrivial projective summands, we have $\operatorname{Im}\left(g_{n}\right) \subseteq J P_{n}$ for all $n$, and thus

$$
\operatorname{Im}\left(g_{k} h_{k-1} g_{k-1} \cdots h_{1} g_{1}\right) \subseteq J^{k} P_{k}=0,
$$

where $J$ is the Jacobson radical and $k$ the Loewy length of $R$. But this contradicts the hypothesis that all finite compositions along the $f_{n}$ 's are nonzero and thus completes the proof.

(b) Matrix subgroups. Start with a column-finite $R$-matrix $A=\left(a_{i j}\right)_{i \in I, j \in J}$, i.e., a matrix $A$ whose entries belong to $R$ such that, for each $j \in J$, the number of nonzero $a_{i j}$ is finite. Such a matrix clearly gives rise to a homogeneous system of linear equations for the elements of an arbitrary left $R$-module, namely the system

$$
\sum_{i \in I} a_{i j} X_{i}=0 \quad \text { for all } j \in J
$$

Given a left $R$-module $M$ and an index $\alpha \in I$, let $[A, \alpha] M$ be the $\alpha$ th projection of the solution set $\mathscr{S} \subseteq M^{I}$ of the above system of equations; in other words,

$$
[A, \alpha] M=\left\{m \in M \mid \text { there exists }\left(m_{i}\right)_{i \in I} \in \mathscr{S} \text { such that } m=m_{\alpha}\right\} .
$$

As is routinely checked, the assignment $R$-Mod $\rightarrow \mathbb{Z}$-Mod, $M \mapsto[A, \alpha] M$, is a subfunctor of the forgetful functor from left $R$-modules to abelian groups. The subgroup $[A, \alpha] M$ of $M$ is called a matrix subgroup; it is called a finite matrix subgroup if $A$ is finite. Note, moreover, that $[A, \alpha] M$ is even an $S$-submodule 
of $M$, whenever $M$ is an $R$-S-bimodule. In particular, each matrix subgroup of $M$ is an $\operatorname{End}_{R}(M)$-submodule of $M$.

Here are a few general types of examples:

- Given any finitely generated right ideal $a$ of $R$, the group $a M$ is a finite matrix subgroup of $M$. $M$.

-The annihilator $\operatorname{Ann}_{M}(X)$ of any subset $X$ of $R$ is a matrix subgroup of

- Any finitely generated $\operatorname{End}_{R}(M)$-submodule of $M$ is a (not necessarily finite) matrix subgroup.

Observe that the matrix subgroups of $M$ form a sublattice of the full lattice of subgroups of $M$; this sublattice is closed under arbitrary intersections, while not closed under infinite sums in general.

For more detailed information on matrix subgroups, see [21 and 23].

Crucial in the present context is the fact that the rings with vanishing left pure global dimension can be characterized via the behavior of the matrix subgroups of their modules.

Theorem I. (See [9 and 21].) An R-module $M$ is $\Sigma$-algebraically compact if and only if $M$ satisfies the descending chain condition for matrix subgroups, equivalently, for finite matrix subgroups. ( $M$ is called $\Sigma$-algebraically compact provided that every direct sum of copies of $M$ is algebraically compact.)

By [16] the algebraically compact modules are precisely the pure injective ones, which yields the notably unpromising observation that $R$ has left pure global dimension zero if and only if every left $R$-module is $(\Sigma$-) algebraically compact. In view of the preceding theorem we thus obtain

Corollary II. The ring $R$ has left pure global dimension zero if and only if each left $R$-module has the descending chain condition for (finite) matrix subgroups.

Our next objective will be to bounce the information on rings with vanishing left pure global dimension, as given in the preceding corollary, to the other side by relating it to the behavior of matrix subgroups of right modules. For that purpose, the following alternate descriptions of matrix subgroups will be helpful.

Lemma 1. For any left $R$-module $M$, the (finite) matrix subgroups of $M$ can be described as follows:

(1) The matrix subgroups of $M$ are precisely the subgroups

$$
\operatorname{Hom}_{R}(P, M)(p)=\left\{f(p) \mid f \in \operatorname{Hom}_{R}(P, M)\right\},
$$

where $P$ is a left $R$-module and $p \in P$. Furthermore, a matrix subgroup of $M$ is finite if and only if it is of the form $\operatorname{Hom}_{R}(P, M)(p)$ for some finitely presented left $R$-module $P$ and $p \in P$. 
(2) (see [9]) The finite matrix subgroups of $M$ coincide with the kernels of the $\mathbb{Z}$-linear maps

$$
M \rightarrow Q \otimes_{R} M, \quad m \mapsto q \otimes m
$$

where $Q$ is a finitely presented right $R$-module and $q \in Q$.

Proof. The verification of (1) is straightforward. For (2) suppose that $Q$ is finitely presented and $q \in Q$. Start with a free resolution

$$
R^{l} \stackrel{h}{\rightarrow} R^{k} \stackrel{g}{\rightarrow} Q \rightarrow 0
$$

such that $g\left(e_{1}\right)=q$, where $e_{1}, \ldots, e_{k}$ is a basis for the right $R$-module $R^{k}$. Moreover, let $f_{1}, \ldots, f_{l}$ be a basis for $R^{l}$, and choose $a_{i j} \in R$ so that $h\left(f_{i}\right)=$ $\sum_{j=1}^{k} e_{j} a_{i j}$ for $i=1, \ldots, l$. In view of the exactness of the sequence

$$
R^{l} \otimes_{R} M \stackrel{h \otimes 1}{\longrightarrow} R^{k} \otimes_{R} M \stackrel{g \otimes 1}{\longrightarrow} Q \otimes_{R} M \rightarrow 0
$$

we obtain the following string of equivalences for each $m \in M: 0=q \otimes m=$ $(g \otimes 1)\left(e_{1} \otimes m\right) \Leftrightarrow$ there are $m_{1}, \ldots, m_{l} \in M$ such that

$$
e_{1} \otimes m=\sum_{i=1}^{l} h\left(f_{i}\right) \otimes m_{i}=\sum_{j=1}^{k} e_{j} \otimes\left(\sum_{i=1}^{l} a_{i j} m_{i}\right) \Leftrightarrow
$$

there are $m_{1}, \ldots, m_{l} \in M$ such that $\sum_{i=1}^{l} a_{i j} m_{i}=\delta_{1 j} m$ for all $j=1, \ldots$, $k \Leftrightarrow m \in[A, 1] M$, where

$$
A=\left(\begin{array}{cccc}
-1 & 0 & \cdots & 0 \\
a_{11} & a_{12} & \cdots & a_{1 k} \\
\vdots & \vdots & & \vdots \\
a_{l 1} & a_{l 2} & \cdots & a_{l k}
\end{array}\right) .
$$

To see the converse, note that each finite matrix subgroup $[B, \beta] M$ of $M$ can be written in the form

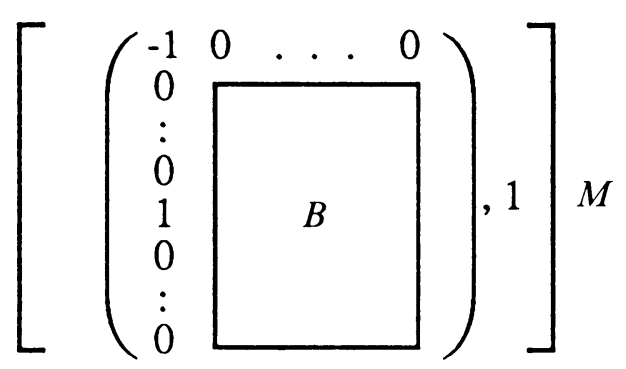

where the first column of the new matrix carries 1 in the row indexed by $\beta$ (we assume that 1 does not occur as a row index of $B$ ). Thus part (2) of the lemma is established. 
(c) "Shifting" matrix subgroups from left to right. A few rather harmless, if technical, computations will show that, given an $R$-S -bimodule $M$ and any injective cogenerator $C$ for Mod- $S$, the lattice of all finite matrix subgroups of the left $R$-module $M$ is anti-isomorphic to the lattice of all finite matrix subgroups of the right $R$-module $\operatorname{Hom}_{S}(M, C)$.

Lemma 2. Suppose that $M$ is an $R$-S-bimodule and $C$ an injective right $S$-module. If $U$ is a finite matrix subgroup of the left $R$-module $M$, then $\operatorname{Hom}_{S}(M / U, C)$ is a finite matrix subgroup of the right $R$-module $\operatorname{Hom}_{S}(M, C)$. Proof. By Lemma 1, Part (1), there exists a finitely presented left $R$-module $P$ together with $p \in P$ such that $U=\operatorname{Hom}_{R}(P, M)(p)$. From the exactness of the sequence

$$
\operatorname{Hom}_{R}(P, M) \stackrel{\varphi}{\rightarrow} M \stackrel{\text { can }}{\longrightarrow} M / U \rightarrow 0,
$$

where $\varphi$ is the evaluation map $\varphi(f)=f(p)$, we deduce exactness of the sequence

$$
0 \rightarrow \operatorname{Hom}_{S}(M / U, C) \rightarrow \operatorname{Hom}_{S}(M, C) \stackrel{\operatorname{Hom}(\varphi, 1)}{\longrightarrow} \operatorname{Hom}_{S}\left(\operatorname{Hom}_{R}(P, M), C\right) .
$$

Next observe that the canonical homomorphism

$$
\sigma: \operatorname{Hom}_{S}(M, C) \otimes_{R} P \rightarrow \operatorname{Hom}_{S}\left(\operatorname{Hom}_{R}(P, M), C\right)
$$

is an isomorphism by our assumptions on $P$ and $C$ (see e.g. [4, Chapter I, $\S 2$, Exercise 14]), and verify that

$$
\sigma^{-1} \circ \operatorname{Hom}(\varphi, 1): \operatorname{Hom}_{S}(M, C) \rightarrow \operatorname{Hom}_{S}(M, C) \otimes_{R} P
$$

is the map which sends $f$ to $f \otimes p$. Thus Part (2) of Lemma 1 yields that $\operatorname{Hom}_{S}(M / U, C)$, being the kernel of the last map, is a finite matrix subgroup of $\operatorname{Hom}_{S}(M, C)$.

We have now smoothed the road to the main point of this subsection, which is to prove

Proposition 3. Let $M$ be an R-S-bimodule, $C$ an injective cogenerator for Mod $-S$ and $M^{+}=\operatorname{Hom}_{S}(M, C)$. Then the lattice of all finite matrix subgroups of the left $R$-module $M$ is anti-isomorphic to the lattice of all finite matrix subgroups of the right $R$-module $M^{+}$via

$$
U \mapsto \operatorname{Ann}_{M^{+}}(U) \text { and } V \mapsto \operatorname{Ann}_{M}(V) .
$$

(Here $\operatorname{Ann}_{M^{+}}(U)=\left\{f \in M^{+} \mid f(m)=0\right.$ for all $\left.m \in U\right\}$ and $\operatorname{Ann}_{M}(V)=\{m \in$ $M \mid f(m)=0$ for all $f \in V\}$.)

Proof. Observe first that, given a finite matrix subgroup $U$ of $M$, Lemma 2 tells us that $\operatorname{Ann}_{M^{+}}(U)=\operatorname{Hom}_{S}(M / U, C)$ is a finite matrix subgroup of $M^{+}$.

To see that $\operatorname{Ann}_{M}(V)$ is a finite matrix subgroup of $M$ if $V$ is a finite matrix subgroup of $M^{+}$, write $V=\operatorname{Hom}_{R}\left(P, M^{+}\right)(p)$, where $P$ is a finitely 
presented right $R$-module and $p \in P$. In view of Lemma 1, part (2), it suffices to show that $\operatorname{Ann}_{M}(V)$ is the kernel of the map

$$
\psi: M \rightarrow P \otimes_{R} M, \quad m \mapsto p \otimes m .
$$

Indeed, since $C$ is a cogenerator for Mod- $S$, we have $p \otimes m=0$ if and only if $g(p \otimes m)=0$ for all $g \in \operatorname{Hom}_{S}\left(P \otimes_{R} M, C\right)$, and in view of the natural isomorphism

$$
\operatorname{Hom}_{S}\left(P \otimes_{R} M, C\right) \cong \operatorname{Hom}_{R}\left(P, M^{+}\right)
$$

we conclude that " $p \otimes m=0$ " is equivalent to " $f(p)(m)=0$ for all $f \in$ $\operatorname{Hom}_{R}\left(P, M^{+}\right)$", that is, to " $h(m)=0$ for all $h \in V$ ".

To check that $\mathrm{Ann}_{M^{+}} \operatorname{Ann}_{M}(V) \subseteq V$, keep the above notation and start with an element $f$ in the left-hand group. Using the fact that $\operatorname{Ann}_{M}(V)=\operatorname{ker} \psi$, we obtain $f(\operatorname{ker} \psi)=0$, i.e., $\operatorname{ker} \psi \subseteq \operatorname{ker} f$, whence there exists a homomorphism $g^{\prime} \in \operatorname{Hom}_{S}(M / \operatorname{ker} \psi, C)$ such that the following diagram commutes:

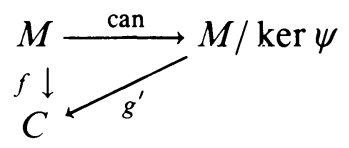

Since $C$ is an injective $S$-module, $g^{\prime}$ can be extended to a map $g \in$ $\operatorname{Hom}_{S}\left(P \otimes_{R} M, C\right)$ via the embedding $M / \operatorname{ker} \psi \rightarrow P \otimes_{R} M$ induced by $\psi$. Thus the diagram

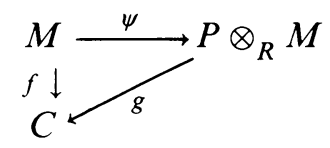

commutes. Referring once more to the canonical isomorphism

$$
\operatorname{Hom}_{S}\left(P \otimes_{R} M, C\right) \cong \operatorname{Hom}_{R}\left(P, M^{+}\right),
$$

we denote the element in $\operatorname{Hom}_{R}\left(P, M^{+}\right)$corresponding to $g$ by $h$, meaning that $h(q)(m)=g(q \otimes m)$ for all $q \in Q$ and $m \in M$. In particular, this yields

$$
h(p)(m)=g(p \otimes m)=g(\psi(m))=f(m)
$$

for all $m \in M$, showing $f=h(p) \in V$ as desired. The inclusion $V \subseteq$ $\operatorname{Ann}_{M^{+}} \operatorname{Ann}_{M}(V)$ is trivial.

The equality $\mathrm{Ann}_{M} \mathrm{Ann}_{M^{+}}(U)=U$ being an immediate consequence of the fact that $C$ is a cogenerator for Mod- $S$, Proposition 3 is established.

(d) Noetherian (artinian) families of homomorphisms. Following Auslander [1], we will call a family $\mathscr{F}$ of homomorphisms between left (right) $R$-modules noetherian if for each concatenation

$$
M_{0} \stackrel{f_{0}}{\rightarrow} M_{1} \stackrel{f_{1}}{\rightarrow} M_{2} \stackrel{f_{2}}{\rightarrow} \cdots
$$

of maps $f_{i}$ in $\mathscr{F}$, there exists an index $n$ such that either $f_{n} \cdots f_{0}=0$ or all maps $f_{k}, k \geq n$, are isomorphisms. 
Dually, call $\mathscr{F}$ artinian provided that, for each concatenation

$$
\ldots \stackrel{f_{2}}{\rightarrow} M_{2} \stackrel{f_{1}}{\rightarrow} M_{1} \stackrel{f_{0}}{\rightarrow} M_{0}
$$

of maps $f_{i}$ in $\mathscr{F}$, either a finite composition of the $f_{i}$ is zero or else all the $f_{k}$ are isomorphisms from a certain point on.

Proposition 4. If $\left(M_{i}\right)_{i \in I}$ is a family of finitely generated indecomposable left $R$-modules such that $\bigoplus_{i \in I} M_{i}$ is $\Sigma$-algebraically compact, the family of all homomorphisms between the $M_{i}$ is noetherian.

Proof. Let $M_{i_{0}} \stackrel{f_{0}}{\rightarrow} M_{i_{1}} \stackrel{f_{1}}{\rightarrow} M_{i_{2}} \stackrel{f_{2}}{\rightarrow} \ldots$ be a sequence of nonisomorphisms, and let $m \in M_{i_{0}}$. Setting $S=\operatorname{End}_{R}\left(\bigoplus_{i \in I} M_{i}\right)$, consider the following chain of matrix subgroups of $\bigoplus_{i \in I} M_{i}$ :

$$
S f_{0}(m) \supseteq S f_{1} f_{0}(m) \supseteq S f_{2} f_{1} f_{0}(m) \supseteq \cdots .
$$

By hypothesis and Theorem I, this sequence becomes stationary, whence there exist an index $k$ and a map $g \in \operatorname{Hom}\left(M_{i_{k+1}}, M_{i_{k}}\right)$ such that

$$
f_{k-1} \cdots f_{0}(m)=g f_{k} f_{k-1} \cdots f_{0}(m) \text {. }
$$

Because $f_{k}$ is a nonisomorphism and $M_{i_{k+1}}$ is indecomposable,

$$
g f_{k} \in \operatorname{Hom}_{R}\left(M_{i_{k}}, M_{i_{k}}\right)
$$

is not an isomorphism. This last ring being local by [23, Theorem 9], the map $1-g f_{k} \in \operatorname{Hom}_{R}\left(M_{i_{k}}, M_{i_{k}}\right)$ is an isomorphism, and from

$$
\left(1-g f_{k}\right) f_{k-1} \cdots f_{0}(m)=0
$$

we therefore deduce $f_{k-1} \cdots f_{0}(m)=0$. But since $M_{i_{0}}$ is finitely generated, we can thus find an index $l$ such that $f_{l} \cdots f_{0}=0$.

The following consequence of Proposition 4 is well known (see [1 and 7]).

Corollary 5. If $R$ has left pure global dimension zero, then

(1) all families of homomorphisms between (finitely generated) indecomposable left $R$-modules are noetherian, and

(2) all families of homomorphisms between finitely presented indecomposable right $R$-modules are artinian.

Proof. Part (1) is an immediate consequence of Corollary II, Proposition 4 and the fact that all indecomposable left $R$-modules are finitely generated. For Part (2) apply the Auslander-Bridger transpose to move from a chain of homomorphisms between finitely presented indecomposable right modules to a reversed chain of homomorphisms between finitely presented indecomposable left modules (see our remarks under part (a) of this section).

\section{MAIN RESUlts}

Based on our new trick of shifting finite matrix subgroups of modules from one side to the other, we will first explore the mirror image of the description of 
vanishing left pure global dimension which we gave in Corollary II. This passage of information from left to right by means of matrix subgroups will be the first step in a game reminiscent of ping-pong; we will then bounce our information across the net several more times via the Auslander-Bridger transpose in order to arrive at the fact that the rings with vanishing left pure global dimension have only finitely many isomorphism types of indecomposable left modules of any fixed finite length; moreover, for each $n$, they have only finitely many isomorphism types of indecomposable finitely presented right modules which are either of length $n$ or $n$-related, i.e., of the form $P / U$ where $P$ is finitely generated projective and $U$ is an $n$-generated submodule of $\operatorname{Rad} P$. (The awkwardness of the statement concerning right $R$-modules is due to the fact that it is still not known whether 'left pure global dimension zero' implies 'right artinian'.)

Theorem 6. (I) The following statements are equivalent for a ring $R$ :

(1) $R$ has left pure global dimension zero.

(2) Each left R-module has the descending chain condition for (finite) matrix subgroups.

(3) Each right $R$-module has the ascending chain condition for finite matrix subgroups.

(II) Moreover, the following are equivalent:

(1) $R$ has left and right pure global dimension zero (i.e., $R$ has finite representation type).

(2) Each left $R$-module has finite length over its endomorphism ring.

(3) Each right $R$-module has finite length over its endomorphism ring.

Proof. (I) The equivalence of (1) and (2) is just Corollary II of $\S 2$. To show “(2) $\Rightarrow(3)$ ", assume (2), and let $N$ be an arbitrary right $R$-module. Since, by hypothesis, the left $R$-module $N^{+}=\operatorname{Hom}_{\mathbb{Z}}(N, \mathbb{Q} / \mathbb{Z})$ has the descending chain condition for finite matrix subgroups, Proposition 3 guarantees the ascending chain condition for finite matrix subgroups of $N$. The proof of " (3) $\Rightarrow(2)$ " is symmetric.

(II) “(1) $\Leftrightarrow(2)$ ". First suppose that (1) holds, and let $M$ be any left $R$ module with endomorphism ring $S$. By part (I), $M$ satisfies the ascending and the descending chain conditions for finite matrix subgroups. The latter condition forces $M$ to be $\Sigma$-algebraically compact in view of Theorem I, which in turn implies that all matrix subgroups of $M$ are finite matrix subgroups: In fact, given an arbitrary matrix subgroup $[A, \alpha] M$ with $A=\left(a_{i j}\right)_{i \in I, j \in J}$ and a finite subset $F$ of the index set $J$, denote by $A(F)$ the finite matrix $\left(a_{i j}\right)_{i \in L, j \in F}$, where $L$ is a finite subset of $I$ containing $\alpha$ such that $a_{i j}=0$ whenever $j \in F$ and $i \in I \backslash L$. By the descending chain condition, the set of all finite matrix subgroups of the form $[A(F), \alpha] M$, where $F$ runs through the finite subsets of $J$, has a minimal element, say $\left[A\left(F_{0}\right), \alpha\right] M$. The latter group clearly contains the original subgroup $[A, \alpha] M$. To see that the two groups coincide, let $m_{\alpha} \in\left[A\left(F_{0}\right), \alpha\right] M$ and observe that by our choice of $F_{0}$ 
the system

$$
\sum_{i \in I \backslash\{\alpha\}} a_{i j} X_{i}=-m_{\alpha} a_{\alpha j} \quad(j \in J)
$$

is finitely solvable and hence globally solvable (use the fact that $M$ is algebraically compact); thus $m_{\alpha} \in[A, \alpha] M$. In particular, every finitely generated $S$-submodule of $M$ is a finite matrix subgroup (keep in mind that every finitely generated $S$-submodule of $M$ is a matrix subgroup for some, in general infinite, matrix; compare $\S 2(\mathrm{~b}))$. Consequently, the $S$-module $M$ satisfies the ascending and the descending chain conditions on finitely generated $S$-submodules, a requirement clearly tantamount to finite length.

Conversely suppose that (2) holds, and let $M$ once more denote a left $R$ module with endomorphism ring $S$. Since every matrix subgroup of $M$ is an $S$-submodule of $M$, our hypothesis endows $M$ with the ascending and the descending chain conditions for matrix subgroups. But by part (I) global validity of the latter conditions for all left $R$-modules means that the left and the right pure global dimension of $R$ vanishes.

The equivalence of (1) and (3) follows by symmetry.

Remark 7. We recover Chase's result [5, Theorem 4.4] as an immediate output of our machinery: If $R$ has left pure global dimension zero, then $R$ is left artinian. In fact, we obtain more sharply that any ring $R$ is left artinian if both $R$ and $\operatorname{Hom}_{\mathbb{Z}}(R, \mathbb{Q} / \mathbb{Z})$ are $\Sigma$-algebraically compact as left $R$-modules. (Just note that by Theorem I, $\Sigma$-algebraic compactness of $R$ as a left $R$-module implies that $R$ is left perfect, while, by Proposition 3, the second hypothesis is equivalent to the ascending chain condition for finite matrix subgroups of $R$, where $R$ is viewed as a right $R$-module; since these subgroups include all finitely generated left ideals, the latter chain condition means that $R$ is left noetherian, and our claim follows.)

As we just saw, the information about solution sets of linear equations which is encapsulated in the condition that the left pure global dimension be zero can be translated into dual data on the right. In order to efficiently exploit the resulting "global" ascending chain condition for finite matrix subgroups, let us first have a look at the condition "locally". Given any right $R$-module $N$ with endomorphism ring $S$, the ascending chain condition for arbitrary $S$ submodules of $N$ clearly implies the ascending chain condition for finite matrix subgroups. While, in general, the converse fails (see the example at the end of this section), it does hold for pure-projective modules, i.e., for direct summands of direct sums of finitely presented modules.

Observation 8. A direct summand of a direct sum of finitely presented modules has the ascending chain condition for finite matrix subgroups precisely if it is noetherian over its endomorphism ring.

Proof. Assume that $N$ is a direct summand of $\bigoplus_{i \in I} N_{i}$, where each $N_{i}$ is a finitely presented right $R$-module, and denote the endomorphism ring of $N$ 
by $S$. Moreover, suppose that $N$ has the ascending chain condition for finite matrix subgroups. We wish to check that every finitely generated $S$-submodule of $N$ is a finite matrix subgroup. Since finite sums of finite matrix subgroups are clearly again of this type, it suffices to show that each cyclic $S$-submodule of $N$ is a finite matrix subgroup of $N$. But if we start with an arbitrary element $x \in N$ and choose a finite subset $F$ of $I$ such that $x \in \bigoplus_{i \in F} N_{i}$, we observe that $S x=\operatorname{Hom}_{R}\left(\bigoplus_{i \in F} N_{i}, N\right)(x)$, and the latter is a finite matrix subgroup of $N$ by Lemma 1 .

This observation makes it easy to glean the crucial piece of information from Theorem 6. Roughly speaking, it says that, while a ring $R$ with vanishing left pure global dimension admits no nonnoetherian family of homomorphisms between its indecomposable left modules (Corollary 5), nonnoetherian families between indecomposable right modules abound, unless $R$ has finite representation type.

Theorem 9. Suppose that $R$ has left pure global dimension zero. Then every direct sum of finitely presented right $R$-modules is noetherian over its endomorphism ring. Moreover:

(I) Given any infinite family $\left(N_{i}\right)_{i \in I}$ of finitely presented right $R$-modules and nonzero elements $n_{i}$ of $N_{i}$, there exist infinitely many distinct indices $i_{0}, i_{1}, i_{2}, \ldots$ in I together with homomorphisms $f_{k}: N_{i_{k-1}} \rightarrow N_{i_{k}}$ such that for all $n \in \mathbb{N}$

$$
f_{n} f_{n-1} \cdots f_{1}\left(n_{i_{0}}\right) \neq 0 \text {. }
$$

In particular: If the $N_{i}$ are pairwise nonisomorphic, then the family of all homomorphisms between the $N_{i}$ 's fails to be noetherian.

(II) Given any infinite family $\left(M_{i}\right)_{i \in I}$ of nonzero finitely generated left $R$ modules, there exist infinitely many pairwise different indices $i_{0}, i_{1}, i_{2}, \ldots$ in I together with homomorphisms $g_{k}: M_{i_{k}} \rightarrow M_{i_{k-1}}$ such that for all $n \in \mathbb{N}$

$$
g_{1} g_{2} \cdots g_{n} \neq 0 \text {. }
$$

(Note that for left modules as for right modules, we are writing homomorphisms on the left.) In particular: If the $M_{i}$ are pairwise nonisomorphic, then the family of all homomorphisms between the $M_{i}$ 's fails to be artinian.

Before we prove Theorem 9, we indicate how it takes us to our first major goal.

Corollary 10. Given a ring $R$ of left pure global dimension zero and any natural number $n$, there are only finitely many isomorphism types of left $R$-modules of length $n$; moreover, the number of types of finitely presented right $R$-modules of length $n$ is finite, and so is the number of types of indecomposable right $R$ modules of the form $P / U$, where $P$ is finitely generated projective and $U$ runs through the n-generated submodules of $\operatorname{Rad} P$.

In particular, $R$ has only countably many isomorphism types of finitely generated (resp. finitely presented) left (resp. right) modules. 
Proof of Corollary 10. Since each of the right or left modules under discussion is a direct sum of indecomposable components, we may restrict our attention to indecomposable modules in the proof of our finiteness claims.

(1) If there were infinitely many isomorphism types of indecomposable left $R$-modules of some fixed length $n$, represented by the family $\left(M_{i}\right)_{i \in I}$ say, then the family of all homomorphisms between them would be nonartinian by part (II) of Theorem 9. But this is impossible by the well-known lemma of Harada and Sai [11, Lemma 11], which says that any composite of $2^{n}-1$ nonisomorphisms between indecomposable modules of length $\leq n$ is zero. This proves our first claim.

(2) The first of our statements concerning right modules can be derived from part (I) of Theorem 9 in an analogous manner. (The additional hypothesis that the modules under consideration be finitely presented reflects our ignorance as to whether $R$ has the DCC for right ideals.) For the second statement, fix $n$ and let $N$ be an indecomposable right module of the form $P / U$ where $P$ is finitely generated projective and $U$ is an $n$-generated submodule of $\operatorname{Rad} P$; without loss of generality, we may assume that $N$ is not itself projective. Then $N$ has a minimal projective resolution $Q \rightarrow P \rightarrow N \rightarrow 0$ where $Q$ is $n$-generated, and the Auslander-Bridger transpose $\operatorname{Tr}(N)$ of $N$ is a factor module of $Q^{*}$. The module $Q$ being a direct summand of the right $R$-module $R^{n}$, the dual $Q^{*}$ is a direct summand of the left $R$-module $R^{n}$, whence $\operatorname{Tr}(N)$ is a factor module of $R^{n}$. But due to the "left-hand part" of our claim-already established-and the fact that $R$ is left artinian-compare Remark 7-there are only finitely many isomorphism types of left modules which are factors of $R^{n}$; using the Auslander-Bridger transpose yet again to bounce this information from left to right, we derive that the number of isomorphism types of indecomposable right modules of the form $P / U$, where $P$ is finitely generated projective and $U$ an $n$-generated submodule of $\operatorname{Rad} P$, is finite as well.

Remark. In [13], Ringel gave an example of a two-sided artinian ring $R$ with infinite center, not of finite representation type, such that for each positive integer $n$ there are at most two indecomposable $R$-modules of length $n$. As is easily verified, the pure global dimension of this ring exceeds zero.

Proof of Theorem 9. For a proof of the first assertion combine Theorem 6(I) with Observation 8.

(I) Now let $\left(N_{i}\right)_{i \in I}$ and $n_{i} \in N_{i}$ be as in our claim, and denote the direct sum of the $N_{i}$ by $N^{(0)}$, its endomorphism ring by $S^{(0)}$. As we have just observed, $N^{(0)}$ is noetherian as an $S^{(0)}$-module. In particular, the $S^{(0)}$-submodule generated by the $n_{i}, i \in I$, can be generated over $S^{(0)}$ by a finite subfamily of the $n_{i}$. Suppose that $\left(n_{i}\right)_{i \in F}$ is such a finite generating set. Then, clearly, there exists an index $i_{0} \in F$ and an infinite subset $I_{1}$ of $I \backslash\left\{i_{0}\right\}$ such that, for each $i \in I_{1}$, we can choose a map $f_{i}^{(1)} \in \operatorname{Hom}_{R}\left(N_{i_{0}}, N_{i}\right)$ with $f_{i}^{(1)}\left(n_{i_{0}}\right) \neq 0$. 
Next let $N^{(1)}$ be the direct sum of the $N_{i}, i \in I_{1}$, let the elements $f_{i}^{(1)}\left(n_{i_{0}}\right) \in$ $N^{(1)}$ for $i \in I_{1}$ take over the role of the $n_{i}$, and recycle the above considerations to obtain an index $i_{1} \in I_{1}$, an infinite subset $I_{2}$ of $I_{1} \backslash\left\{i_{1}\right\}$ and homomorphisms $f_{i}^{(2)} \in \operatorname{Hom}_{R}\left(N_{i_{1}}, N_{i}\right)$ such that $f_{i}^{(2)} f_{i_{1}}^{(1)}\left(n_{i_{0}}\right) \neq 0$ for all $i \in I_{2}$. An obvious iteration of this procedure yields distinct indices $i_{0}, i_{1}, i_{2}, \ldots$ in $I$ and maps

$$
f_{i_{k}}^{(k)}: N_{i_{k-1}} \rightarrow N_{i_{k}}
$$

such that $f_{i_{k}}^{(k)} \cdots \cdots f_{i_{1}}^{(1)}\left(n_{i_{0}}\right) \neq 0$ for all $k \geq 1$, which completes the proof of part (I).

To show part (II), let $\left(M_{i}\right)_{i \in I}$ be an infinite family of finitely generated (=finitely presented) left $R$-modules. If almost all of the $M_{i}$ are projective, our claim is obvious. Otherwise discard the projective members of the family and split off a maximal projective direct summand from each of the remaining $M_{i}$ (consult $\S 2$ (a) for more detail). Without loss of generality we may thus assume that none of the $M_{i}$ has a projective direct summand. Next we apply part (I) to the family $\left(\operatorname{Tr}\left(M_{i}\right)\right)_{i \in I}$ of the corresponding Auslander-Bridger transposes to obtain distinct indices $i_{0}, i_{1}, i_{2}, \ldots$ in $I$ and connecting homomorphisms $f_{k}: \operatorname{Tr}\left(M_{i_{k-1}}\right) \rightarrow \operatorname{Tr}\left(M_{i_{k}}\right)$ such that all the compositions $f_{n} f_{n-1} \cdots f_{1}, n \in \mathbb{N}$, are nonzero. By the comments we made in $\S 2(\mathrm{a})$, the maps $f_{k}$ give rise to maps

$$
g_{k}=\operatorname{Tr}\left(f_{k}\right): \operatorname{Tr}\left(\operatorname{Tr}\left(M_{i_{k}}\right)\right) \cong M_{i_{k}} \rightarrow \operatorname{Tr}\left(\operatorname{Tr}\left(M_{i_{k-1}}\right)\right) \cong M_{i_{k-1}} .
$$

Since by construction none of the modules $\operatorname{Tr}\left(M_{i_{k}}\right)$ has a nonzero projective summand, Lemma $\operatorname{Tr}$ of $\S 2$ (a) provides us with an index $m$ such that all strings $g_{m} \cdots g_{n}$ with $n>m$ are nonzero. Reindex if necessary to arrive at our claim.

As we pointed out in the introduction, it is well known that vanishing of the left and right pure global dimension implies finite representation type. Fuller and Reiten [7] derived this result from a theorem of Auslander's [1] which in turn makes heavy use of Auslander's characterization of rings of finite type via the functor category Funct (mod $-R, \mathrm{Ab}$ ), the latter being the category of functors from finitely generated $R$-modules to abelian groups. We obtain the result at stake as an immediate consequence of Theorem 9 and the very elementary Proposition 4.

Corollary 11. Suppose that $R$ has left pure global dimension zero, and let $\left(M_{i}\right)_{i \in I}$ be a family of representatives of the finitely generated indecomposable left $R$-modules. If the right $R$-module $\bigoplus_{i \in I} \operatorname{Tr}\left(M_{i}\right)$ is $\Sigma$-algebraically compact, then $R$ has finite representation type. In particular, every ring with vanishing left and right pure global dimension is of finite representation type.

Proof. If the set $I$ were infinite, then the family of all homomorphisms between the right $R$-modules $\operatorname{Tr}\left(M_{i}\right)$ would be nonnoetherian by Theorem 9 . On the other hand, $\Sigma$-algebraic compactness of $\bigoplus_{i \in I} \operatorname{Tr}\left(M_{i}\right)$ excludes this possibility (Proposition 4). 
We digress from our main track to record the following by-product of our investigation.

Corollary 12. Suppose that $R$ is an Artin algebra and that $\left(M_{i}\right)_{i \in I}$ is a family of finitely generated indecomposable left $R$-modules.

(I) If $\bigoplus_{i \in I} M_{i}$ is $\Sigma$-algebraically compact, then, given any natural number $n$, there are only finitely many pairwise nonisomorphic summands $M_{i}$ of length at most $n$.

(II) If $C$ denotes the center of $R$ and $\mathscr{D}$ is the Morita duality

$$
\mathscr{D}: R-\bmod \rightarrow \bmod -R, \quad X \mapsto \operatorname{Hom}_{C}(X, E(C / \operatorname{rad} C)),
$$

where $E(C / \operatorname{Rad} C)$ is the injective hull of $C / \operatorname{rad} C$, then the following statements are equivalent:

(1) Both $\bigoplus_{i \in I} M_{i}$ and $\bigoplus_{i \in I} \mathscr{D}\left(M_{i}\right)$ are $\Sigma$-algebraically compact $R$-modules.

(2) The number of isomorphism types of $M_{i}$ 's $(i \in I)$ is finite.

Proof. Our claim is really a corollary of Proposition 3, Observation 8 and the proofs of Theorem 9 and Corollary 10.

(I) Combining Theorem I and Proposition 3 yields: $\bigoplus_{i \in I} M_{i}$ is $\Sigma$-algebraically compact if and only if the direct product $\prod_{i \in I} \mathscr{D}\left(M_{i}\right)$ has the ascending chain condition on finite matrix subgroups. Since passage to matrix subgroups commutes with direct products and direct sums, $\prod_{i \in I} \mathscr{D}\left(M_{i}\right)$ has the ascending chain condition on finite matrix subgroups if and only if the sum $\bigoplus_{i \in I} \mathscr{D}\left(M_{i}\right)$ does, which means that the latter is noetherian over its endomorphism ring by Proposition 8. Thus, Part (I) is an immediate consequence of the arguments we used to prove Theorem 9 and Corollary 10 (keep in mind that, since $\mathscr{D}$ is a Morita duality for the ring $R$, the length of $M$ equals the length of $\mathscr{D}(M)$ for every finitely generated $R$-module $M$ ).

(II) That (2) implies (1) is clear in view of the fact that each $M_{i}$ is of finite length over its endomorphism ring. For the converse, assume that (1) holds, and suppose that there are infinitely many pairwise nonisomorphic candidates among the $M_{i}$; say $\left(M_{j}\right)_{j \in J}$ is such an infinite subfamily without repetitions. By Proposition 3, $\Sigma$-algebraic compactness of $\bigoplus_{j \in J} \mathscr{D}\left(M_{j}\right)$ is equivalent to the ascending chain condition for finite matrix subgroups of $\bigoplus_{j \in J} M_{j}$, whence, using once more Observation 8 and the proof of Theorem 9, we see that the family of all homomorphisms between the $M_{j}$ 's is nonnoetherian. But by Proposition 4, this is incompatible with $\Sigma$-algebraic compactness of $\bigoplus_{j \in J} M_{j}$, and the proof is complete.

In particular, we derive from Corollary 12 that, over any Artin algebra, an uncountable direct sum of finitely generated indecomposable modules, which are pairwise nonisomorphic, can never be $\Sigma$-algebraically compact.

We conclude with an example of a module $M$ which enjoys the ascending chain condition for finite matrix subgroups without being noetherian over its endomorphism ring. 
Example 13. Starting with a field $F$, let $R$ be the factor ring

$$
F\left[X_{i}: i \in \mathbb{N}\right] /\left(X_{i}: i \in \mathbb{N}\right)^{2} \text {, }
$$

where the $X_{i}$ are independent commuting indeterminates over $F$. By [23], $R$ is $\Sigma$-algebraically compact as an $R$-module. If $E=E(R / J)$ denotes the minimal injective cogenerator over $R$ (here $J$ denotes the Jacobson radical of $R$ ), then, by Proposition 3, the $R$-module $E \cong \operatorname{Hom}_{R}(R, E)$ has the ascending chain condition for finite matrix subgroups.

On the other hand, the module $E$ is not noetherian over its endomorphism ring, because, in view of the equality $A=\operatorname{Ann}_{R} \operatorname{Ann}_{E}(A)$ for every ideal $A$ of $R$, we could otherwise derive the descending chain condition for ideals of $R$; but the latter chain condition is clearly violated.

Note added in proof. Our main result (Corollary 10) was independently proved via model theory by M. Prest, J. London Math. Soc. 38 (1988), 403-409.

\section{REFERENCES}

1. M. Auslander, Representation theory of Artin algebras. II, Comm. Algebra 1 (1974), 293310.

2. __ Large modules over Artin algebras, Algebra, Topology and Categories, Academic Press, New York, 1976.

3. M. Auslander and M. Bridger, Stable module theory, Mem. Amer. Math. Soc. No. 94 (1969).

4. N. Bourbaki, Commutative algebra, Hermann, Paris, 1972.

5. S. U. Chase, Direct products of modules, Trans. Amer. Math. Soc. 97 (1960), 457-473.

6. I. S. Cohen and I. Kaplansky, Rings for which every module is a direct sum of cyclic modules, Math. Z. 54 (1951), 97-101.

7. K. R. Fuller and I. Reiten, Note on rings of finite representation type and decompositions of modules, Proc. Amer. Math. Soc. 50 (1975), 92-94.

8. P. A. Griffith, On the decomposition of modules and generalized left uniserial rings, Math. Ann. 184 (1970), 300-308.

9. L. Gruson and C. U. Jensen, Modules algébriquement compacts et foncteurs $\lim ^{(i)}$, C. R. Acad. Sci. Paris Ser. A 276 (1973), 1651-1653.

10. __ Deux applications de la notion de L-dimension, C. R. Acad. Sci. Paris Ser. A 282 (1976), 23-24.

11. M. Harada and Y. Sai, On categories of indecomposable modules. I, Osaka J. Math. 7 (1970), 323-344.

12. G. Koethe, Verallgemeinerte abelsche Gruppen mit hyperkomplexem Operatorenring, Math. Z. 39 (1935), 31-44.

13. C. M. Ringel, Representations of $K$-species and bimodules, J. Algebra 41 (1976), 269-302.

14. C. M. Ringel and H. Tachikawa, $Q F-3$ rings, J. Reine Angew. Math. 272 (1975), 49-72.

15. S. O. Smalø, The inductive step of the second Brauer-Thrall conjecture, Canad. J. Math. 32 (1978), 342-349.

16. R. B. Warfield, Jr., Purity and algebraic compactness for modules, Pacific J. Math. 28 (1969), 699-719.

17. _ Rings whose modules have nice decompositions, Math. Z. 125 (1972), 187-192.

18. __ Serial rings and finitely presented modules, J. Algebra 37 (1975), 187-222.

19. M. Zayed, Indecomposable modules over right pure semisimple rings, Monatsh. Math. 105 (1988), 165-170. 
20. W. Zimmermann, Einige Charakterisierungen der Ringe ueber denen reine Untermoduln direkte Summanden sind, Bayer. Akad. Wiss. Math.-Natur. Kl. S.-B. 1972, Abt. II (1973), 77-79.

21. __ Rein-injektive direkte Summen von Moduln, Comm. Algebra 5 (1977), 1083-1117.

22. B. Zimmermann-Huisgen, Rings whose right modules are direct sums of indecomposable modules, Proc. Amer. Math. Soc. 77 (1979), 191-197.

23. B. Zimmermann-Huisgen and W. Zimmermann, Algebraically compact rings and modules, Math. Z. 161 (1978), 81-93.

Department of Mathematics, University of California, Santa Barbara, California 93106

Mathematisches Institut der Universitaet Muenchen, Theresienstrasse 39,8000 Muenchen 2, West Germany 